ISSN: $2394-2258$

Available at http://scientificadvances.co.in

DOI: http://dx.doi.org/10.18642/ijamml_7100121626

\title{
TWO CHARACTERIZATIONS OF SWITCHED NONLINEAR SYSTEMS WITH AVERAGE DWELL TIME
}

\author{
Jiqiang Wang ${ }^{1}$, Zhongzhi Hu${ }^{2}$, Hong Yue ${ }^{3}$ \\ and Georgi Dimirovski ${ }^{4}$ \\ ${ }^{1,2}$ Jiangsu Province Key Laboratory of Aerospace Power Systems, College of \\ Energy and Power Engineering, Nanjing University of Aeronautics and \\ Astronautics, 29 Yudao Street, Nanjing 210016, P. R. China \\ ${ }^{3}$ Department of Electronic \& Electrical Engineering, University of Strathclyde, \\ Glasgow, UK \\ ${ }^{4}$ Dogus University of Istanbul, Istanbul 34722, Turkey
}

\begin{abstract}
This note aims to establish the fast switching condition with average dwell time satisfying an upper bound. Important results are obtained on the behaviour of switched nonlinear dynamical systems. In specific, this note contributes in the following three aspects: (1) establish the condition of fast switching of switched nonlinear systems; (2) obtain the condition of arbitrary switching stability of switched nonlinear dynamical systems using a weak Lyapunov functions approach; and (3) prove the necessity of the average dwell time condition associated with the conventional multiple Lyapunov functions' framework.
\end{abstract}

\footnotetext{
${ }^{*}$ Corresponding author.

E-mail address: jiqiang.wang@nuaa.edu.cn (Jiqiang Wang).
}

Copyright (c) 2016 Scientific Advances Publishers 2010 Mathematics Subject Classification: 93D05.

Submitted by Li Li.

Received January 21, 2016; Revised March 19, 2016 
Keywords: switched nonlinear systems, multiple Lyapunov function, average dwell time, arbitrary switching stability.

\section{Introduction}

It is well-known that the behaviour of hybrid systems may have markedly different properties from each of their component, e.g., one switched system can be stable although all of its components are unstable, while an unfortunate switching signal may destabilize the switched system even though all of its components are stable. Most of the existing literature concerns the problem of stability under arbitrary switching and many important results have been obtained during the past decades (Morse [9]; Johansson \& Rantzer [6]; Liberzon [7]; Hespanha [4]; Sun \& Ge [11]). To guarantee stability under arbitrary switching, the common Lyapunov function method plays an important role. This is because the existence of a common Lyapunov function implies the global uniform asymptotic stability of the switched system. The importance of common Lypunov function is consolidated by a converse theorem, dictating that if the switched system is globally uniformly asymptotically stable, then all the subsystems share a common Lyapunov function (Molchanov \& Pyatnitskiy [8]).

Recently, multiple Lyapunov functions and the associated dwell time or average dwell time are recognized as another efficient tool to analyse stability (Branicky [2]; Hespanha \& Morse [5]). The concept of average dwell time switching introduced in (Hespanha \& Morse [5]) is more general than dwell time switching in stability analysis and related control design problems (Hespanha [4]; Sun et al. [10]; Vu et al. [12]). It implies that the number of switches in a finite interval is bounded from above, and the average time between consecutive switching is not less than a constant (Zhang \& Gao [14]). The multiple Lyapunov function approach is believed to reduce conservatism than the common Lyapunov function method. In fact, when specialized to linear systems, some well- 
known design procedures for explicit construction of multiple Lyapunov functions have been developed, e.g., the $S$-procedure (Boyd et al. [1]) and the hysteresis switching (Liberzon [7]; Wicks et al. [13]). In multiple Lyapunov functions approaches, it is generally assumed that each Lyapunov-like function associated for each subsystem is decreasing with time. Allowing the Lyapunov-like function to rise to a limited extent is considered in (Ye et al. [3]). This is an interesting extension as it is intuitively appealing that may further reduce the conservatism than usual multiple Lyapunov functions, nevertheless than the common Lyapunov function approach. This observation forms the foundation of the paper, aiming to show how far it can proceed along this line of idea. In specific, it will be shown that both slow switching and fast switching can be studied within this framework; the implications are then worked out producing important results in the theory of stability of switched nonlinear systems. These collective results form the contribution of the paper.

Notation. The notation used in this paper is fairly standard. $\mathfrak{R}^{N}$ represents the $N$-dimensional Euclidean space; $C^{1}$ denotes the space of continuously differentiable functions; a function $k:[0, \infty] \rightarrow[0, \infty]$ is called class $K_{\infty}$ if it is continuous, strictly increasing and unbounded with $k(0)=0$.

\section{Preliminaries}

The concept of average dwell time is defined below:

Definition (Hespanha \& Morse [5]). For a switching signal $\sigma$ and any $t_{2}>t_{1}>t_{0}$, let $N_{\sigma}\left(t_{1}, t_{2}\right)$ be the number of switching over the interval $\left[t_{1}, t_{2}\right)$. If the condition $N_{\sigma}\left(t_{1}, t_{2}\right) \leq N_{0}+\left(t_{2}-t_{1}\right) / \tau_{a}$ holds for $N_{0} \geq 1$, $\tau_{a}>0$, then $N_{0}$ and $\tau_{a}$ are called the average dwell time (ADT) and the chatter bound, respectively. 
Then the following important result can be obtained:

Theorem 1 (Hespanha \& Morse [5]). Consider the switched system (1), and let $\alpha$ and $\mu$ be given constants. Suppose that there exist smooth functions $V_{\sigma(t)}: \Re^{N} \rightarrow \Re, \sigma(t) \in \ell$, and two $K_{\infty}$ functions $k_{1}$ and $k_{2}$ such that for each $\sigma(t)=i$, the following conditions hold: $k_{1}\left(\left|x_{t}\right|\right) \leq$ $V_{i}\left(x_{t}\right) \leq k_{2}\left(\left|x_{t}\right|\right), \dot{V}_{i}\left(x_{t}\right) \leq-\alpha V_{i}\left(x_{t}\right)$, and for any $(i, j) \in \ell \times \ell, i \neq j$, $V_{i}\left(x_{t}\right) \leq \mu V_{j}\left(x_{t}\right)$; then the system is globally uniformly asymptotically stable for any switching signal with $A D T \tau_{a}>\tau_{a}^{*}=\frac{\ln \mu}{\alpha}$.

Theorem 1 considers multiple Lyapunov functions with "jump" on switching boundary. An extension due to (Ye et al. [3]) and further exposed in (Zhang \& Gao [14]) allows the Lyapunov-like function to rise to a limited extent, in addition to the jump on switching boundary. This is the so-called week Lyapunov functions, and it allows both the jump on the switching boundary and the increase over any interval. Now consider $\sigma(t)=i$ and within the interval $\left[t_{i}, t_{i+1}\right)$, denote the unions of scattered subintervals during which the week Lyapunov function is increasing and decreasing by $T_{r}\left(t_{i}, t_{i+1}\right)$ and $T_{d}\left(t_{i}, t_{i+1}\right)$, respectively. Hence $\left[t_{i}, t_{i+1}\right)=T_{r}\left(t_{i}, t_{i+1}\right) \cup T_{d}\left(t_{i}, t_{i+1}\right)$. Further use $T_{r}\left(t_{i+1}-t_{i}\right)$ and $T_{d}\left(t_{i+1}-t_{i}\right)$ to represent the length of $T_{r}\left(t_{i}, t_{i+1}\right)$ and $T_{d}\left(t_{i}, t_{i+1}\right)$ correspondingly. Then the following important result can be obtained:

Theorem 2 (Ye et al. [3]; Zhang \& Gao [14]). Consider the switched system $\dot{x}_{t}=f_{\sigma}\left(x_{t}\right)$, and let $\alpha>0, \beta>0$ and $\mu>1$ are prescribed constants. If there exist smooth functions $V_{\sigma(t)}: \mathfrak{R}^{n} \rightarrow \mathfrak{R}$ and two $K_{\infty}$ functions $k_{1}$ and $k_{2}$ such that for each $\sigma(t)=i$, the following conditions hold:

$$
\begin{gathered}
k_{1}\left(\left|x_{t}\right|\right) \leq V_{i}\left(x_{t}\right) \leq k_{2}\left(\left|x_{t}\right|\right), \\
\dot{V}_{i}\left(x_{t}\right) \leq \begin{cases}-\alpha V_{i}\left(x_{t}\right) & \text { over } \quad t \in T_{d}\left(t_{i}, t_{i+1}\right), \\
\beta V_{i}\left(x_{t}\right) & \text { over } t \in T_{r}\left(t_{i}, t_{i+1}\right),\end{cases}
\end{gathered}
$$




$$
V_{i}\left(x_{t}\right) \leq \mu V_{j}\left(x_{t}\right) \quad \forall\left(\sigma(t)=i \quad \& \quad \sigma\left(t^{-}\right)=j\right),
$$

then the system is GUAS for any switching signal with $A D T$

$$
\tau_{a}>\tau_{a}^{s}=\frac{(\alpha+\beta) T_{\max }+\ln ^{\mu}}{\alpha}, \quad T_{\max }=\max T_{r}\left(t_{i-1}, t_{i}\right), \quad \forall i .
$$

It is seen that the result above actually includes Theorem 1 as a special case, e.g., $\beta=0$ implies no increase over the interval and hence $T_{\max }=0$, then the ADT condition reduces to the ADT condition $\tau_{a}>\tau_{a}^{*}=\frac{\ln \mu}{\alpha}$ in Theorem 1. It is the generality of the weak Lyapunov functions that motives us to derive a fast switching rule below.

\section{Main Results}

Theorem 2 is a slow switching result in the sense that it is characterized by a lower bound on ADT. We shall now derive a fast switching result providing an upper bound on ADT.

Theorem 3. Consider the switched system $\dot{x}_{t}=f_{\sigma}\left(x_{t}\right)$, and let $\alpha>0, \beta>0$ and $\mu>1$ are prescribed constants. If there exist smooth functions $V_{\sigma(t)}: \Re^{n} \rightarrow \mathfrak{R}$ and two $K_{\infty}$ functions $k_{1}$ and $k_{2}$ such that for each $\sigma(t)=i$, the following conditions hold:

$$
\begin{gathered}
k_{1}\left(\left|x_{t}\right|\right) \leq V_{i}\left(x_{t}\right) \leq k_{2}\left(\left|x_{t}\right|\right), \\
\dot{V}_{i}\left(x_{t}\right) \leq \begin{cases}-\alpha V_{i}\left(x_{t}\right) \quad \text { over } \quad t \in T_{d}\left(t_{i}, t_{i+1}\right), \\
\beta V_{i}\left(x_{t}\right) \quad \text { over } \quad t \in T_{r}\left(t_{i}, t_{i+1}\right),\end{cases} \\
V_{i}\left(x_{t}\right) \leq \mu V_{j}\left(x_{t}\right) \quad \forall\left(\sigma(t)=i \quad \& \quad \sigma\left(t^{-}\right)=j\right),
\end{gathered}
$$

then the system is GUAS for any switching signal with ADT

$$
\tau_{a}<\tau_{a}^{f}=\frac{(\alpha+\beta) T_{\min }-\ln ^{\mu}}{\beta}, \quad T_{\min }=\min T_{d}\left(t_{i-1}, t_{i}\right), \quad \forall i
$$


Proof. For $t \in\left[t_{i}, t_{i+1}\right)$, we have

$$
\begin{aligned}
& V_{i}\left(x_{t}\right) \leq e^{-\alpha T_{d}\left(t_{i}, t\right)+\beta T_{r}\left(t_{i}, t\right)} V_{i}\left(x_{t^{-}}\right) \\
& \leq e^{-\alpha T_{d}\left(t_{i}, t\right)-\beta T_{d}\left(t_{i}, t\right)+\beta T_{d}\left(t_{i}, t\right)+\beta T_{r}\left(t_{i}, t\right)} V_{i}\left(x_{t^{-}}\right) \\
& \leq e^{\beta\left(t-t_{i}\right)} e^{-(\alpha+\beta) T_{d}\left(t_{i}, t\right)} V_{i}\left(x_{t^{-}}\right) \\
& \leq e^{\beta\left(t-t_{i}\right)} e^{-(\alpha+\beta) T_{\min }} \mu V_{i-1}\left(x_{t^{+}}\right) \\
& \leq e^{\beta\left(t-t_{0}\right)}\left(e^{-(\alpha+\beta) T_{\min }}\right)^{N_{\sigma}\left(t_{0}, t\right)} \mu^{N_{\sigma}\left(t_{0}, t\right)} V_{0}\left(x_{t_{0}}\right) \\
& \left.\leq e^{\left\{N_{\sigma}\left(t_{0}, t\right)\left[\ln ^{\mu}-(\alpha+\beta) T_{\min }\right]+\beta\left(t-t_{0}\right)\right.}\right\} V_{0}\left(x_{t_{0}}\right),
\end{aligned}
$$

where we have made the definition $T_{\min } \equiv \min _{l} T_{d}\left(t_{i}-t_{i-1}\right)$, that is the minimum decreasing interval over any switching sequence. Hence if $N_{\sigma}\left(t_{0}, t\right)\left[\ln ^{\mu}-(\alpha+\beta) T_{\min }\right]+\beta\left(t-t_{0}\right)<0$, then $V_{i}\left(x_{t}\right)$ will be decreasing and the system will achieve GUAS. Now the condition $N_{\sigma}\left(t_{0}, t\right)$ $\left[\ln ^{\mu}-(\alpha+\beta) T_{\min }\right]+\beta\left(t-t_{0}\right)<0 \quad$ is exactly the average dwell time defined by $\tau_{\alpha} \equiv \frac{t-t_{0}}{N_{\sigma}\left(t_{0}, t\right)}$. This completes the proof.

Theorem 3 is very interesting as it can be combined with Theorem 2 to prove a beautiful theorem stating that the average dwell time condition is "if and only if " and is thus an important progress in the stability theory of switched nonlinear systems. The fundamental idea comes from the following simple observation: a system will achieve arbitrary switching stability if the upper bound for fast switching is larger than the lower bound for slow switching. But before proving the result, let us first work on the implications of the fast and slow switching conditions. And then the proof will become "evident". 
Case 1. $\mu=1$

Then the fast and slow switching conditions reduce to

$$
\tau_{a}>\tau_{a}^{s}=\frac{(\alpha+\beta) T_{\max }}{\alpha}, \quad T_{\max }=\max T_{r}\left(t_{i-1}, t_{i}\right), \quad \forall i,
$$

and

$$
\tau_{a}<\tau_{a}^{f}=\frac{(\alpha+\beta) T_{\min }}{\beta}, \quad T_{\min }=\min T_{d}\left(t_{i}-t_{i-1}\right), \quad \forall i \in \ell .
$$

Hence if the value of the fast switching is greater than that of the slow switching, that is, $\tau_{a}^{s} \leq \tau_{a}^{f}$, or $\frac{T_{\max }}{T_{\min }} \leq \frac{\alpha}{\beta}$, then the system will be GUAS under any switching signals. The situation here is that the minimum decreasing should be larger than the maximum increasing of the energy function over any interval. In the extreme case, that is $\beta=0$, which also implies $T_{\max }=0$, then the slow switching becomes $\tau_{a}^{s}=0$ while the fast switching is $\tau_{a}^{f}=\infty$. The system is GUAS under arbitrary switching! This actually becomes the case of usual multiple Lyapunov functions with exact matching on the switching boundary. To summarize, we have the following result:

Theorem 4. Consider the switched system $\dot{x}_{t}=f_{\sigma}\left(x_{t}\right)$, and let $\alpha>0$, $\beta>0$ are prescribed constants. If there exist smooth functions $V_{\sigma(t)}: \mathfrak{R}^{n} \rightarrow \Re$ and two $K_{\infty}$ functions $k_{1}$ and $k_{2}$ such that for each $\sigma(t)=i$, the following conditions hold:

$$
\begin{gathered}
k_{1}\left(\left|x_{t}\right|\right) \leq V_{i}\left(x_{t}\right) \leq k_{2}\left(\left|x_{t}\right|\right), \\
\dot{V}_{i}\left(x_{t}\right) \leq \begin{cases}-\alpha V_{i}\left(x_{t}\right) \quad \text { over } \quad t \in T_{d}\left(t_{i}, t_{i+1}\right), \\
\beta V_{i}\left(x_{t}\right) \quad \text { over } t \in T_{r}\left(t_{i}, t_{i+1}\right),\end{cases} \\
V_{i}\left(x_{t}\right)=V_{j}\left(x_{t}\right) \quad \forall\left(\sigma(t)=i \quad \& \quad \sigma\left(t^{-}\right)=j\right),
\end{gathered}
$$

then the system is GUAS for arbitrary switching signal if the following condition holds: 
$\frac{T_{\max }}{T_{\min }} \leq \frac{\alpha}{\beta}$, where $T_{\max } \equiv \max T_{r}\left(t_{i-1}, t_{i}\right)$ and $T_{\min } \equiv \min T_{d}\left(t_{i-1}, t_{i}\right), \quad \forall i$.

Proof. The correctness of the result is proved by collecting the above arguments.

Case 2. $\beta=0$

The case $\beta=0$ means no increasing over any interval and hence $T_{\max }=0$. Then the slow and fast switching conditions become

$$
\tau_{a}>\tau_{a}^{s}=\frac{\ln ^{\mu}}{\alpha}
$$

and

$$
\tau_{a}^{f}=\infty \& T_{\min } \geq \frac{\ln ^{\mu}}{\alpha}, \quad T_{\min }=\min T_{d}\left(t_{i}-t_{i-1}\right), \quad \forall i \in \ell
$$

That is, the slow switching condition reduces to the ADT condition defined in (Hespanha \& Morse [5]), while the fast switching rule implies that the minimum dwell time required for arbitrary switching stability with ADT is exactly the limit $\frac{\ln ^{\mu}}{\alpha}$. This simple observation leads to the following important result:

Theorem 5. Consider the switched system $\dot{x}_{t}=f_{\sigma}\left(x_{t}\right)$ and let $\alpha>0$, $\mu>1$ be given constants. Suppose that there exist $C^{1}$ functions $V_{\sigma(t)}: \mathfrak{R}^{N} \rightarrow \Re, \sigma(t) \in \ell$, and two $K_{\infty}$ functions $k_{1}$ and $k_{2}$ such that $\forall \sigma(t)=i, k_{1}\left(\left|x_{t}\right|\right) \leq V_{i}\left(x_{t}\right) \leq k_{2}\left(\left|x_{t}\right|\right), \dot{V}_{i}\left(x_{t}\right) \leq-\alpha V_{i}\left(x_{t}\right)$, and $\forall(i, j) \in \ell \times \ell$, $i \neq j, V_{i}\left(x_{t}\right) \leq \mu V_{j}\left(x_{t}\right)$, then the system is GUAS for any switching signal if and only if the ADT satisfies the condition $\tau_{a}>\tau_{a}^{*}=\frac{\ln \mu}{\alpha}$. 
Proof. The sufficiency part has already been shown in Theorem 1 and we show the necessity part below. We do this by first considering the fast switching rule in Theorem 3. Then the above discussion has shown that the minimum dwell time among all the switching sequences is exactly $\tau_{a}^{*}=\frac{\ln \mu}{\alpha}$. This implies that the ADT condition $\tau_{a}>\tau_{a}^{*}$ is in fact tight. That is, to guarantee the system to be GUAS for any switching signal, the ADT has to satisfy $\tau_{a}>\tau_{a}^{*}$, hence the necessity of the ADT condition is proved. To further explain the result without referring to the special cases discussed here, we proceed with another angle to look at the problem.

Consider $\beta=0$, that is, over any interval $\left[t_{i}, t_{i+1}\right)$ the Lyapunov-like function $V_{i}\left(x_{t}\right)$ is non-increasing, then Theorem 3 tells that the only requirement for fast switching stability is the nominator $(\alpha+\beta) T_{\min }-\ln ^{\mu} \geq 0$, that is, $T_{\min } \geq \frac{\ln ^{\mu}}{\alpha}$. It can then deduce from the definition $T_{\min } \equiv \min T_{d}\left(t_{i}-t_{i-1}\right), \forall i \in \ell$ that the minimum decreasing duration over any interval should satisfy $T_{\min } \geq \tau^{*}=\frac{\ln ^{\mu}}{\alpha}$. This is equivalently to say that the minimum dwell time over any switching sequence should be at least $\tau^{*}=\frac{\ln ^{\mu}}{\alpha}$.

Now the case $\beta=0$ is exactly the Lyapunov-like functions defined in Theorem 5. To recap, the sufficiency part says that the system is GUAS for any switching signal if the ADT satisfies the condition $\tau_{a}>\tau_{a}^{*}=\frac{\ln \mu}{\alpha} ;$ the analysis here shows that to guarantee GUAS, the minimum dwell time over any switching sequence should be at least $\tau^{*}=\frac{\ln ^{\mu}}{\alpha}$. That is the estimation $\tau_{a}>\tau_{a}^{*}=\frac{\ln \mu}{\alpha}$ is actually tight, demonstrating the necessity of the ADT condition. 
Case 3. $\alpha=0$

The case $\alpha=0$ implies no decreasing over any interval, hence $T_{\min }=0$. The two switching inequalities now reduce to $\tau_{a}>\infty$ and $\tau_{a}<0$, which is obviously trivial. Hence no switching sequence exists to stabilize the system at all, conforming with the intuitive result.

\section{Conclusion}

This note has characterized the constrained switching of switched dynamical systems through slow switching and fast switching. These two types of switching provide low and upper bounds for stability of switched nonlinear systems. Of particular interest is the fast switching rule to guarantee GUAS and the condition for arbitrary switching stability of switched nonlinear dynamical systems. Based on this fundamental result, a series of important results have been obtained that provide information and insight into switched systems. Finally, the note has proved that the usual ADT condition associated with multiple Lyapunov functions is in fact IFF. These three results are the main contribution of the note. Based on the results, the effect of time delay and nonlinearity such as saturation on stabilizing property can be analysed. This is left for further investigation.

\section{Funding Acknowledgement}

We are grateful for the financial support of the Natural Science Foundation of Jiangsu Province (No. BK20140829); and the Fundamental Research Funds for the Central Universities (No. NS2016024). 


\section{References}

[1] S. P. Boyd, L. El Ghaoui, E. Feron and V. Balakrishnan, Linear Matrix Inequalities in Systems and Control Theory, SIAM Studies in Applied Mathematics, Vol. 15, SIAM, Philadelphia, 1994.

[2] M. S. Branicky, Multiple Lyapunov functions and other analysis tools for switched and hybrid systems, IEEE Transactions on Automatic Control 43(4) (1998), 475-482.

[3] H. Ye, A. N. Michel and L. Hou, Stability theory for hybrid dynamical systems, IEEE Transactions on Automatic Control 43(4) (1998), 461-474.

[4] J. P. Hespanha, Uniform stability of switched linear systems extensions of Lassalle's invariance principle, IEEE Transactions on Automatic Control 49(4) (2004), 470-482.

[5] J. P. Hespanha and A. S. Morse, Stability of switched systems with average dwell time, Proceedings of 38th Conference on Decision and Control, Phoenix, (1999), 26552660 .

[6] M. Johansson and A. Rantzer, Computation of piecewise quadratic Lyapunov functions for hybrid systems, IEEE Transactions on Automatic Control 43(4) (1998), 555-559.

[7] D. Liberzon, Switching in Systems and Control, Boston: Birkhäuser, 2003.

[8] A. P. Molchanov and Y. S. Pyatnitskiy, Criteria of asymptotic stability of differential and difference inclusions encountered in control theory, Systems and Control Letters 13 (1989), 59-64.

[9] A. S. Morse, Supervisory control of families of linear set-point controllers: Part 1: Exact matching, IEEE Transactions on Automatic Control 41(10) (1996), 1413-1431.

[10] X. Sun, J. Zhao and D. Hill, Stability and L2-gain analysis for switched delay systems: A delay-dependent method, Automatica 42(10) (2006), 1769-1774.

[11] Z. Sun and S. S. Ge, Analysis and synthesis of switched linear control systems, Automatica 41(2) (2005), 181-195.

[12] L. Vu, D. Chatterjee and D. Liberzon, Input-to-state stability of switched systems and switching adaptive control, Automatica 43(4) (2007), 639-646.

[13] M. A. Wicks, P. Peleties and R. A. Decarlo, Construction of piecewise Lyapunov functions for stabilizing switched systems, Proceedings of the $33^{\text {rd }}$ Conference on Decision and Control, pp. 3492-3497.

[14] L. Zhang and H. Gao, Asynchronously switched control of switched linear systems with average dwell time, Automatica 46 (2010), 953-958. 